\title{
Association between frailty and 30-day outcomes after discharge from hospital
}

\author{
Sharry Kahlon MD MHA, Jenelle Pederson BA, Sumit R. Majumdar MD MPH, Sara Belga MD, \\ Darren Lau MD PhD, Miriam Fradette BScPharm, Debbie Boyko RN, Jeffrey A. Bakal PhD, Curtis Johnston MD, \\ Raj S. Padwal MD MSc, Finlay A. McAlister MD MSc
}

CMAJ Podcasts: author interview at soundcloud.com/cmajpodcasts/150100-res

\begin{abstract}
Background: Readmissions after hospital discharge are common and costly, but prediction models are poor at identifying patients at high risk of readmission. We evaluated the impact of frailty on readmission or death within 30 days after discharge from general internal medicine wards.
\end{abstract}

Methods: We prospectively enrolled patients discharged from 7 medical wards at 2 teaching hospitals in Edmonton. Frailty was defined by means of the previously validated Clinical Frailty Scale. The primary outcome was the composite of readmission or death within 30 days after discharge.

Results: Of the 495 patients included in the study, $162(33 \%)$ met the definition of frailty: $91(18 \%)$ had mild, 60 (12\%) had moderate, and $11(2 \%)$ had severe frailty. Frail patients were older, had more comorbidities, lower quality of life, and higher LACE scores at discharge than those who were not frail. The composite of 30-day readmission or death was higher among frail than among nonfrail patients (39 [24.1\%] v. 46 [13.8\%]). Although frailty added additional prognostic information to predictive models that included age, sex and LACE score, only moderate to severe frailty $(31.0 \%$ event rate) was an independent risk factor for readmission or death (adjusted odds ratio 2.19, 95\% confidence interval 1.12-4.24).

Interpretation: Frailty was common and associated with a substantially increased risk of early readmission or death after discharge from medical wards. The Clinical Frailty Scale could be useful in identifying high-risk patients being discharged from general internal medicine wards.
$\mathrm{R}$ eadmissions within 30 days after hospital discharge are common and costly occurrences. Although many studies have attempted to identify patients at highest risk of readmission, neither experienced clinicians nor experienced researchers using rigorously developed administrative data-rich algorithms can accurately predict which patients will not successfully transition back into the community. ${ }^{1-6}$ This suggests that currently unrecognized factors likely play a major role in readmission risk. Identification of these factors would be important for future initiatives to reduce readmission rates by targeting resources to those at highest risk.

Frailty is a frequently underdiagnosed condition, with prevalence estimates ranging from $27 \%$ to $80 \%$ among inpatients ${ }^{7-9}$ and from $4 \%$ to $59 \%$ among older adults living in the community ${ }_{10}^{10}$ depending on the frailty measure used and the population evaluated. Frailty is a multidimensional syndrome of decreased reserve and resistance to stressors leading to increased vulnerability to adverse outcomes. ${ }^{11-14}$ The 2 models of frailty most commonly used in the literature are the phenotype model (e.g., the approach proposed by Fried and colleagues, ${ }^{15}$ which is based on 5 objective variables assessed at one point in time that do not include psychosocial and cognitive variables) and the cumulative deficit model (e.g., the Clinical Frailty Index, which is based on a mix of more than 30 variables capturing function in many domains over time). ${ }^{16-18}$

Although the gold standard for frailty assessment is a comprehensive geriatric assessment by a multidisciplinary team, both the phenotype and cumulative deficit models appear reasonably accurate for identifying frailty. However, both are somewhat cumbersome for routine use at the bedside. ${ }^{12}$ For these reasons, the Clinical Frailty Scale was developed and relies on clinical judgment based on history taking and clinical examination. The Clinical Frailty Scale is easy to ad-
Competing interests: None declared.

This article has been peer reviewed.

Accepted: May 5, 2015

Online: May 25, 2015

Correspondence to:

Finlay McAlister,

finlay.mcalister@ualberta.ca

CMAJ 2015. DOI:10.1503/ cmaj.150100 
minister at the bedside; has been used by physicians, allied health professionals and research assistants; does not require any special equipment; is highly correlated with the Fried frailty index $(r=0.8) ;{ }^{17}$ and appears to be valid, reliable and reproducible. ${ }^{19}$ Some risk-prediction models, such as the LACE Index, have tried to incorporate frailty, but they did not find it to be a significant independent variable, possibly owing to the frailty measure used. A systematic review of 30 risk-prediction models for hospital readmission found that only 2 included functional status. ${ }^{4}$

We conducted a study to evaluate whether frailty identified using the Clinical Frailty Scale is an independent predictor of death or readmission within 30 days after discharge from hospital.

\section{Methods}

\section{Study design and population}

This was a prospective cohort study. We enrolled patients 18 years of age and older who were being discharged from 7 general internal medicine wards at 2 teaching hospitals in Edmonton (University of Alberta and Royal Alexandra hospitals) between October 2013 and November 2014. Patients were excluded if they did not live in the province, had severe cognitive impairment (5 or more errors on the Short Portable Mental Status Questionnaire, on the assumption their ability to answer questionnaires accurately would be compromised), ${ }^{20}$ were deemed by their attending physician to have foreshortened life expectancy that would preclude 90-day follow-up, or were transferred to or from a long-term care facility, another inpatient service or another acute care hospital. If a patient was admitted more than once during the study period, we collected data from the first admission. The follow-up period was 30 days after discharge.

All participants provided informed consent. The study design was approved by the Health Research Ethics Board at the University of Alberta.

\section{Determination of frailty}

Frailty was defined using the Clinical Frailty Scale derived from the Canadian Study on Health and Aging. This well-validated assessment tool is based on clinical judgment and quantifies frailty on a scale of 1 (very fit) to 9 (terminally ill). ${ }^{17}$ Trained research assistants aware of the study hypothesis determined the Clinical Frailty Scale scores by interviewing participants and reviewing each participant's hospital chart. They assigned the score based on the patient's (or proxy's) selfreported best status in the week before becoming ill with the reason for the index hospital admission. We trained our research assistants to calibrate their frailty assessments with those of experienced clini- cians, and scores were reproducible between examiners. The Clinical Frailty Scale has been previously used by our team. ${ }^{19}$

We considered patients to be frail if their Clinical Frailty Scale score was 5 or higher (which represents mild, moderate, severe or very severe frailty and is consistent with published cut-off points ${ }^{17,19}$ ). Of note, mild frailty (score of 5) corresponds to difficulty with 1 or more instrumental activities of daily living (e.g., finances, shopping, meal preparation, housework, medications); moderate frailty (score of 6) corresponds to difficulty with 1 or 2 activities of daily living (e.g., bathing, dressing, climbing stairs); and severe frailty (score of 7 or 8 ) refers to being completely dependent for 3 or more activities of daily living, regardless of whether the cause is physical or cognitive.

\section{Outcome measures}

The primary outcome measure was the composite of readmission (to any acute care hospital in the province) or death within 30 days after discharge from the index hospital. Data on these outcomes were collected by study coordinators through telephone interviews with the patients and review of their electronic health records (Alberta Netcare records) 30 days after discharge; the coordinators were unaware of the study hypothesis and the frailty assessments done at discharge. Secondary outcomes included each element of the primary outcome individually, and the frequency of emergency department visits within the 30-day follow-up period.

\section{Other covariates}

The research assistants collected data on the participants' sociodemographic characteristics, medical comorbidities and medications from the patients' self-reports, the audits of their hospital charts and electronic health records, and contact with patients' pharmacies. They also captured data on length of stay and calculated each patient's LACE score, which is currently the best risk-prediction model for outcomes within 30 days after discharge. ${ }^{1}$ (LACE is a nmemonic derived from variables independently associated with the outcome of death or urgent readmission within the first month after discharge: length of stay ["L"]; acuity of the admission ["A"]; comorbidity of the patient, as measured with the Charlson Comorbidity Index score ["C"']; and emergency department use, measured as the number of visits in the 6 months before admission ["E"].)

\section{Statistical analysis}

We compared characteristics and outcomes of patients according to frailty status at the time of discharge using the $t$ test for continuous variables 
and the $\chi^{2}$ test for categorical variables. We used multivariable logistic regression analysis, adjusting for age and sex, to determine the association between frailty and the composite outcome of readmission or death. We used the HosmerLemeshow goodness-of-fit test to assess model fit. To determine whether frailty provided additional prognostic information above the LACE Index, we compared models with and without the LACE Index using the $\mathrm{C}$ statistic and the Integrated Discrimination Improvement index. All analyses were performed with the use of SAS software (SAS Institute, Inc.); $p$ values of less than 0.05 were considered to be statistically significant.

\section{Results}

Of 1147 potentially eligible patients discharged alive from the medical wards during the study period, 498 were enrolled in our study; 3 patients withdrew, which left 495 (99.4\%) with 30-day follow-up data (Figure 1). The mean age was 64 years, $252(50.6 \%)$ were female, the vast majority (485 [97.4\%]) were admitted via the emergency department, and the median number of comorbidities was 5 (interquartile range 3-7). The top 5 reasons for hospital admission were heart failure $(n=50)$, pneumonia $(n=50)$, chronic obstructive pulmonary disorder (COPD, $n=38$ ), urinary tract infection $(n=27)$ and acute complications of diabetes $(n=26)$. Of note, 163 patients (32.7\%) had diabetes.

Of the 495 patients, $162(32.7 \%)$ were considered to be frail (Clinical Frailty Scale score $\geq 5$ ) in the week before becoming unwell (91 had mild, 60 moderate and 11 severe frailty). Compared with nonfrail patients, frail patients were older, were more likely to be female, had more comorbidities, had more hospital admissions in the year before enrolment, were less likely to live independently before and after hospital admission, had lower scores on the EuroQol Health Questionnaire (a standardized measure of health-related quality of life [www.euroqol.org]) and had slower gait speeds (as determined by the timed get-up-and-go test) (Table 1). Serum albumin levels and mean scores on depression and anxiety did not differ between frail and nonfrail patients, nor did the proportion of patients discharged from teaching wards (Table 1).

Overall, 85 patients were readmitted or died within 30 days after discharge (Table 2), with the most responsible diagnoses being congestive heart failure $(n=11)$, pneumonia $(n=8), \operatorname{COPD}(n=6)$, malignant disease $(n=5)$ and renal failure $(n=4)$. Frail patients had higher event rates than nonfrail patients $(24.1 \%$ v. $13.8 \%$; age- and sex-adjusted odds ratio [OR] 2.01, 95\% confidence interval [CI] 1.19-3.41). Frailty as defined by the Clinical
Frailty Scale (which included mild frailty) was not independently associated with the composite outcome of readmission or death when we adjusted for age, sex and LACE score (adjusted OR 1.52, 95\% CI 0.87-2.62). Patients with moderate or severe frailty $(n=71)$ were more likely than nonfrail patients to be readmitted or to die within 30 days after discharge $(31.0 \%$ [22/71] v. $13.8 \%$; OR adjusted for age and sex 3.19, 95\% CI 1.70-6.00; OR adjusted for age, sex and LACE score 2.19, 95\% 1.12-4.24). The Integrated Discrimination Improvement index was statistically significant for all models that incorporated frailty in addition to age, sex and LACE score $(p<0.001$ for each comparison), which indicated that there was an improvement in the ability of the model both to predict which patients will have an event and to predict which will not. When we looked at frailty as a continuous measure, we found an increased risk of 30-day readmission or death associated with each additional point on the Clinical Frailty Scale, even after we adjusted for age, sex and LACE score (adjusted OR 1.35, 95\% CI 1.111.64), but this was driven largely by the very high risk among patients with a frailty score of 7 or 8 (adjusted OR 9.92, 95\% CI 2.40-40.0).

Frail patients were more likely than nonfrail patients to present to an emergency department during the 30-day follow-up period $(32.7 \% \mathrm{v}$.

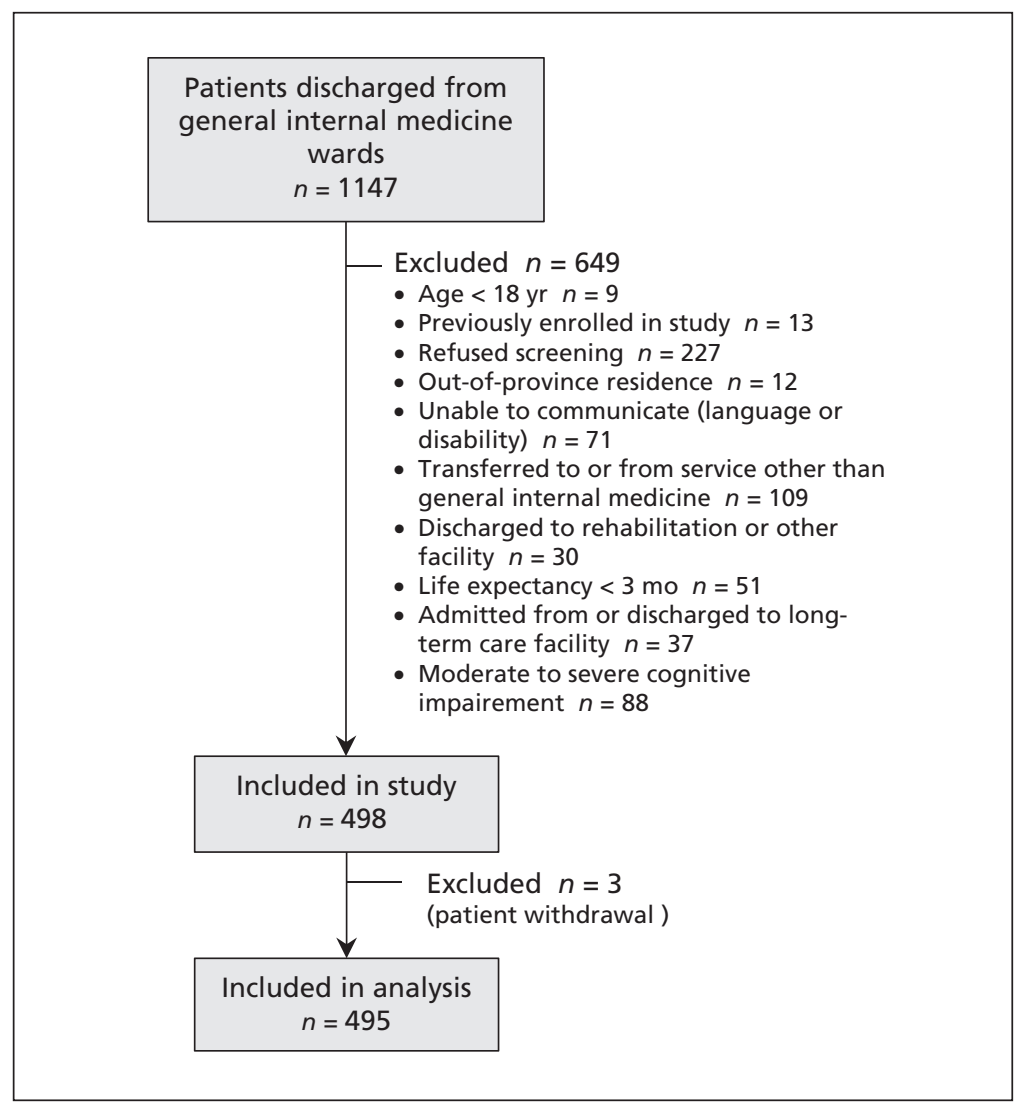

Figure 1: Selection of patients for the study cohort. 
Table 1: Baseline characteristics of patients discharged from hospital who were included in the study

\begin{tabular}{|c|c|c|c|}
\hline \multirow[b]{2}{*}{ Characteristic } & \multicolumn{2}{|c|}{ Frailty status; mean $(95 \% \mathrm{Cl}) *$} & \multirow[b]{2}{*}{$p$ valuet } \\
\hline & $\begin{array}{c}\text { Frail } \\
\text { (CFS score > 4) } \\
n=162\end{array}$ & $\begin{array}{c}\text { Not frail } \\
\text { (CFS score } \leq 4) \\
n=333\end{array}$ & \\
\hline Age, yr, mean $\pm S D$ & $72.5 \pm 15.0$ & $58.2 \pm 18.4$ & $<0.001$ \\
\hline Female sex & $105(64.8)$ & $145(43.5)$ & $<0.001$ \\
\hline No. of comorbidities & $6.3(5.8-6.7)$ & $4.2(3.9-4.5)$ & $<0.001$ \\
\hline $\begin{array}{l}\text { Charlson Comorbidity } \\
\text { Index score }\end{array}$ & $3.6(3.3-3.9)$ & $2.4(2.2-2.6)$ & $<0.001$ \\
\hline $\begin{array}{l}\text { No. of hospital admissions } \\
\text { in past } 12 \mathrm{mo}\end{array}$ & $1.2(1.0-1.4)$ & $0.6(0.5-0.8)$ & $<0.001$ \\
\hline Discharged from teaching ward & $125(77.2)$ & $264(79.3)$ & 0.6 \\
\hline $\begin{array}{l}\text { Living situation before index } \\
\text { admission }\end{array}$ & & & $<0.001$ \\
\hline Living at home independently & $43(26.5)$ & $246(73.9)$ & \\
\hline Living at home with help & $91(56.2)$ & $78(23.4)$ & \\
\hline Assisted-living facility $\ddagger$ & $28(17.3)$ & $9(2.7)$ & \\
\hline EQ-5D overall score & $59.7(56.5-62.9)$ & $65.2(63.3-67.1)$ & 0.002 \\
\hline EQ-5D mobility self-report & & $n=331$ & $<0.001$ \\
\hline No problems & $13(8.0)$ & $191(57.7)$ & \\
\hline Some problems & $132(81.5)$ & $139(42.0)$ & \\
\hline Confined to bed & $17(10.5)$ & $1(0.3)$ & \\
\hline EQ-5D self-care report & & $n=332$ & $<0.001$ \\
\hline No problems & $70(43.2)$ & $284(85.5)$ & \\
\hline Some problems & $82(50.6)$ & $48(14.5)$ & \\
\hline Unable to wash or dress self & $10(6.2)$ & $0 \quad(0.0)$ & \\
\hline PHQ-9 score & $7.9(7.0-8.8)$ & $7.1(6.5-7.7)$ & 0.1 \\
\hline GAD-2 score & $1.8(1.5-2.1)$ & $1.6(1.4-1.8)$ & 0.2 \\
\hline Goals of care in hospital & $n=148$ & $n=319$ & $<0.001$ \\
\hline Resuscitation and ICU & $70(47.3)$ & $267(83.7)$ & \\
\hline ICU but no resuscitation & $33(22.3)$ & $22(6.9)$ & \\
\hline No ICU, no resuscitation & $45(30.4)$ & $29(9.1)$ & \\
\hline Comfort care & $0 \quad(0.0)$ & $1(0.3)$ & \\
\hline Timed get-up-and-go test, s & $24.4(21.7-27.1)$ & $13.1(11.8-14.3)$ & $<0.001$ \\
\hline BMI & $31.4(29.5-33.4)$ & $27.8(27.1-28.6)$ & $<0.001$ \\
\hline eGFR before discharge & $65.6(61.0-70.3)$ & $80.4(77.21-83.6)$ & $<0.001$ \\
\hline Serum albumin level & $34.0(32.7-35.3)$ & 33.7 (32.5-34.9) & 0.8 \\
\hline $\begin{array}{l}\text { No. of prescription } \\
\text { medications at discharge }\end{array}$ & $8.5 \quad(8.0-9.0)$ & $5.3(5.0-5.7)$ & $<0.001$ \\
\hline LACE score§ at discharge & $12.9(12.5-13.3)$ & $10.9(10.6-11.2)$ & $<0.001$ \\
\hline \multicolumn{4}{|c|}{$\begin{array}{l}\text { Note: } \mathrm{BMI}=\text { body mass index, CFS = Clinical Frailty Scale, } \mathrm{Cl}=\text { confidence interval, eGFR = } \\
\text { estimated glomerular filtration rate, EQ-5D = EuroQol Health Questionnaire (maximum score } \\
100 \text { ), GAD-2 = Generalized Anxiety Disorder } 2 \text { Questionnaire, ICU = intensive care unit, } \\
\text { PHQ-9 = Patient Health Questionnaire, SD = standard deviation. } \\
\text { *Unless otherwise indicated. } \\
\text { tThe } t \text { test was used for continuous variables and the } \chi^{2} \text { test for categorical variables. } \\
\text { fIncludes independent seniors' apartments with provision of meals with or without } \\
\text { supervised medication consumption or on-site nursing support, but not nursing homes. } \\
\text { SLACE is a nmemonic derived from variables independently associated with the outcome of } \\
\text { death or urgent readmission within the first month after discharge: length of stay ["L"]; acuity } \\
\text { of the admission ["A"]; comorbidity of the patient, as measured with the Charlson Comorbidity } \\
\text { Index score ["C"]; and emergency department use, measured as the number of visits in the } \\
6 \text { months before admission ["E"]. }\end{array}$} \\
\hline
\end{tabular}

23.4\%; age- and sex-adjusted OR 1.75, 95\% CI 1.10-2.76), but the 2 groups had similar patterns of outpatient physician visits (mean 1.85 v. 1.82; age- and sex-adjusted $p$ value $=0.8$ ). Similar to differences observed before the index admission, frail patients were significantly less likely than nonfrail patients to be discharged home to live independently (16.5\% v. $66.4 \%)$ and significantly more likely to be discharged home with home care $(62.2 \%$ v. $30.7 \%)$ or discharged to an assisted-living facility $(21.3 \%$ v. $3.0 \%)(p<$ 0.001 for each comparison).

\section{Interpretation}

There are 3 key findings from our study. First, frailty was common among patients being discharged from the medical wards, even among patients being discharged home. Second, frailty was associated with an increased risk of readmission or death within 30 days after discharge and increased use of health services even after we adjusted for age and sex. Finally, the presence of moderate to severe frailty added prognostic information that improved the ability to predict rates of readmission or death beyond the LACE score, currently the best risk-prediction model.

Our findings are consistent with those from previous studies showing that frailty was common and associated with poorer outcomes after discharge. However, most of the previous studies were limited because of a lack of clinical data, variable definitions of frailty and poor generalizability owing to data being drawn from atypical or narrow patient populations, or because they did not evaluate whether assessment of frailty improved existing readmission risk-prediction rules. ${ }^{13,14,19,21,22}$ Mirroring our findings, a recent report from the US Health and Retirement Study found that only patients who were dependent for at least 1 activity of daily living (corresponding to our definition of moderate to severe frailty) were at increased risk of readmission; however, that study used reports of functional status 430 days (median) before the index hospital admission. ${ }^{23}$ Although cumulative deficit models such as the Clinical Frailty Scale appear to predict risk of death better than phenotype models, ${ }^{24}$ we explored the frailty scale's ability to predict 30-day outcomes after discharge. The extent to which various frailty instruments overlap in their ability to predict the risk of readmission is unknown and is the subject of other research by our group.

Our finding that moderate to severe frailty as defined by the Clinical Frailty Scale added prognostic information beyond that available from the LACE Index is important for clinicians, policymakers and researchers. The LACE Index has been the best means available using administra- 
Table 2: Outcomes within 30 days after discharge, by frailty status

\begin{tabular}{|lccccc|} 
& & & \multicolumn{3}{c|}{ OR for frailty $(95 \% \mathrm{Cl})$ * } \\
\cline { 3 - 6 } Outcome $\leq 30 \mathrm{~d}$ & $\begin{array}{c}\text { Frail } \\
n=162\end{array}$ & $\begin{array}{c}\text { Not frail } \\
n=333\end{array}$ & Crude & $\begin{array}{c}\text { Adjusted for } \\
\text { age and sex }\end{array}$ & $\begin{array}{c}\text { Adjusted for age, } \\
\text { sex and LACE scoret }\end{array}$ \\
\hline Readmission or death & $39(24.1)$ & $46(13.8)$ & $1.98(1.23-3.18)$ & $2.01(1.19-3.41)$ & $1.52(0.87-2.62)$ \\
\hline Readmission & $36(22.2)$ & $45(13.5)$ & $1.82(1.13-2.97)$ & $1.90(1.11-3.26)$ & $1.42(0.81-2.49)$ \\
\hline Death & $7(4.3)$ & $6(1.8)$ & $2.46(0.81-7.45)$ & $2.18(0.65-7.39)$ & $1.33(0.37-4.76)$ \\
\hline $\begin{array}{l}\text { Visit to emergency } \\
\text { department }\end{array}$ & $53(32.7)$ & $78(23.4)$ & $1.59(1.05-2.41)$ & $1.75(1.10-2.76)$ & $1.43(0.68-2.30)$ \\
\hline $\begin{array}{l}\text { Note: Cl }=\text { confidence interval, OR }=\text { odds ratio. } \\
\text { *All models showed nonsignificance on the Hosmer-Lemeshow goodness-of-fit test. } \\
\text { tSee Table 1 for explanation of LACE Index. }\end{array}$ & & & \\
\hline
\end{tabular}

tive data by which to identify patients at highest risk of readmission or death after discharge. However, it is imperfect, with a $\mathrm{C}$ statistic of 0.68 in its initial validation study ${ }^{1}$ and 0.67 in our study. Use of the simple Clinical Frailty Scale in addition to the LACE Index significantly improved the latter's accuracy and discrimination in our study. This also makes sense clinically, because frailty as defined by the Clinical Frailty Scale has been previously shown to be an independent predictor of length of stay, in-hospital mortality and new placement in a long-term care facility. ${ }^{25}$

Although we focused on frailty in this study, it is only part of the picture for inpatients in general internal medicine services. Numerous studies have shown that hospital admission itself plays a role in subsequent functional deterioration, particularly if patients have prolonged periods of bed rest or cannot take food by mouth during the index admission. ${ }^{26}$ Loss of independence, decreased functional ability and restricted activity all seem to bear poorly on recovery after discharge. ${ }^{27-30}$ Although frailty or vulnerability before becoming ill may affect outcomes after discharge, patients in hospital may also experience an acquired, transient period of risk for adverse events that is harmful in addition to the stress of the acute illness. This "posthospital syndrome" is a multidimensional construct that incorporates sleep deprivation, cognitive stress, poor nutrition and physical pain. ${ }^{26}$ Patients who are already frail before hospital admission may be more sensitive to the stresses of this syndrome and at higher risk of readmission and poor outcomes. This is likely one of the reasons for the low proportion of readmitted patients whose most responsible diagnosis is the same as the one during their first hospital admission. ${ }^{31}$

\section{Limitations}

Although this was a prospective cohort study with objective assessment of outcomes by observers unaware of the patients' frailty scores, the study has some limitations. First, we did not evaluate the functional status of patients after hospital discharge. Changes in functional status are likely to be even more frequent than readmission or death. Second, we included patients discharged from general medical wards at 2 sites (an inner-city hospital and a university campus hospital); therefore, results may not be generalizable to patients admitted for nonmedical reasons, to nonteaching hospitals or in other geographic areas. However, studies involving other inpatient populations have shown similar results to ours. ${ }^{13,19,21-23}$ Third, although we were able to determine whether patients were discharged with home care services in place, we could not tell if frail patients received more home care in hours per day than nonfrail patients, and any discrepancy in the provision of home care could have mitigated differences in outcomes between the groups. Fourth, our eligibility criteria decreased the range of the Clinical Frailty Scale (i.e., exclusion of patients with foreshortened life expectancy and those from long-term care facilities resulted in few patients with very high frailty scores) and, therefore, decreased the likelihood of showing an association between frailty and death or readmission within 30 days after discharge. Lastly, the frailty assessment, based on a clinician's overall impression of each patient's premorbid function, may be seen as subjective. However, we have previously shown the Clinical Frailty Scale to be valid and reproducible between examiners at the same hospitals included in this study. ${ }^{19}$

\section{Conclusion}

Frailty defined using the Clinical Frailty Scale was common and was an independent predictor of readmission or death within 30 days after discharge from medical wards. However, even with this additional prognostic information, risk prediction remains imperfect. Further research is needed to identify additional predictors of early readmission or death after discharge. 


\section{References}

1. van Walraven C, Dhalla IA, Bell C, et al. Derivation and validation of an index to predict early death or unplanned readmission after discharge from hospital to the community. CMAJ 2010;182:551-7.

2. van Walraven $\mathrm{C}$, Bennett $\mathrm{C}$, Jennings $\mathrm{A}$, et al. Proportion of hospital readmissions deemed avoidable: a systematic review. CMAJ 2011;183:E391-402.

3. Gruneir A, Dhalla IA, van Walraven C, et al. Unplanned readmissions after hospital discharge among patients identified as being at high risk for readmission using a validated predictive algorithm. Open Med 2011;5:e104-11.

4. Kansagara D, Englander H, Salanitro A, et al. Risk prediction models for hospital readmission: a systematic review. JAMA 2011;306:1688-98.

5. Allaudeen N, Schnipper JL, Orav EJ, et al. Inability of providers to predict unplanned readmissions. J Gen Intern Med 2011;26:771-6.

6. Cotter PE, Bhalla VK, Wallis SJ, et al. Predicting readmissions: poor performance of the LACE index in an older UK population. Age Ageing 2012;41:784-9.

7. Joosten E, Demuynck M, Detroyer E, et al. Prevalence of frailty and its ability to predict in hospital delirium, falls and 6-month mortality in hospitalized older patients. BMC Geriatr 2014;14:1.

8. Hilmer SN, Perera V, Mitchell QS, et al. The assessment of frailty in older people in acute care. Australas J Ageing 2009;28:182-8.

9. Wou F, Gladman JFR, Bradshaw L, et al. The predictive properties of frailty-rating scales in the acute medical unit. Age Ageing 2013;42:776-81.

10. Collard RM, Boter H, Schoevers RA, et al. Prevalence of frailty in community-dwelling older persons: a systematic review. J Am Geriatr Soc 2012;60:1492-9.

11. Fried LP, Ferrucci L, Darer J, et al. Untangling the concepts of disability, frailty and comorbidity: implications for improved targeting and care. J Gerontol A Biol Sci Med Sci 2004;59:255-63.

12. Clegg A, Young J, Iliffe S, et al. Frailty in elderly people. Lancet 2013;381:752-62.

13. McAdams-DeMarco MA, Law A, Salter ML. Frailty and early hospital readmission after kidney transplant. Am J Transplant 2013;13:2091-5.

14. Makary MA, Segev DL, Pronovost PJ, et al. Frailty as a predictor of surgical outcomes in older patients. J Am Coll Surg 2010;210:901-8.

15. Fried LP, Tangen CM, Walston J, et al. Frailty in older adults: evidence for a phenotype. J Gerontol A Biol Sci Med Sci 2001; 56:M146-56.

16. Song X, Mitnitski A, Rockwood K. Prevalence and 10-year outcomes of frailty in older adults in relation to deficit accumulation. J Am Geriatr Soc 2010;58:681-7.

17. Rockwood K, Song X, Macknight C, et al. A global clinical measure of fitness and frailty in elderly people. CMAJ 2005 173:489-95.

18. Mitnitski AB, Mogilner AJ, Rockwood K. Accumulation of deficits as a proxy measure of aging. ScientificWorldJournal 2001;1:323-36.

19. Bagshaw SM, Stelfox HT, McDermid MD, et al. Association between frailty and short- and long-term outcomes among critically ill patients: a multicentre prospective cohort study. CMAJ 2014;186:E95-102.

20. Pfeiffer E. A short portable mental status questionnaire for the assessment of organic brain deficit in elderly patients. $J \mathrm{Am}$ Geriatr Soc 1975;23:433-41.

21. Robinson TN, Wu DS, Pointer L, et al. Simple frailty score predicts postoperative complications across surgical specialities. Am J Surg 2013;206:544-50.
22. Dai YT. WU SC, Weng R. Unplanned hospital readmission and its predictors in patients with chronic conditions. J Formos Med Assoc 2002; 101:779-85.

23. Greysen SR, Cenzer IS, Auerbach AD, et al. Functional impairment and hospital readmission in Medicare seniors. JAMA Intern Med 2015; 175:559-65.

24. Kulminski AM, Ukraintseva SV, Kulminskaya IV, et al. Cumulative deficits better characterize susceptibility to death in elderly people than phenotypic frailty: lessons from the Cardiovascular Health Study. J Am Geriatr Soc 2008;56:898-903.

25. Basic D, Shanely C. Frailty in an older inpatient population: using the Clinical Frailty Scale to predict patient outcomes. J Aging Health 2015;27:670-85.

26. Krumholz HM. Post-hospital syndrome - an acquired, transient condition of generalized risk. N Engl J Med 2013;368:100-2.

27. Boyd CM, Landefeld CS, Counsell SR, et al. Recovery of activities of daily living in older adults after hospitalization for acute medical illness. J Am Geriatr Soc 2008;56:2171-9.

28. Gill TM, Allore HG, Holford TR, et al. Hospitalization, restricted activity and the development of disability among older persons. JAMA 2004;292:2115-24.

29. Gill TM, Williams CS, Tinetti ME. The combined effects of baseline vulnerability and acute hospital events on the development of functional dependence among community living older persons. J Gerontol A Biol Sci Med Sci 1999;54:M377-83.

30. Brown CJ, Roth DL, Allman RM. Trajectories of life-space mobility after hospitalization. Ann Intern Med 2009;150:372-8.

31. Dharmarajan K, Hsieh AF, Lin Z, et al. Diagnoses and timing of 30 day readmissions after hospitalizations for heart failure acute Myocardial infarction or pneumonia. JAMA 2013;309:355-63.

Affiliations: Division of General Internal Medicine (Kahlon, Pederson, Majumdar, Belga, Lau, Padwal, McAlister); Patient Health Outcomes Research and Clinical Effectiveness Unit (Bakal, McAlister); Epidemiology Coordinating and Research (EPICORE) Centre (Fradette, Boyko, McAlister), University of Alberta; Department of Medicine (Johnston), Royal Alexandra Hospital, Edmonton, Alta.

Contributors: Finlay McAlister, Sumit Majumdar and Raj Padwal contributed to the conception and design of the study and obtained funding for the study. Finlay McAlister, Sumit Majumdar and Curtis Johnston supervised the study. Sharry Kahlon, Jenelle Pederson, Sara Belga, Darren Lau, Miriam Fradette and Debbie Boyko contributed to the acquisition of data; Jeffrey Bakal was responsible for data analysis. Sharry Kahlon and Finlay McAlister drafted the manuscript. All of the authors critically revised the manuscript, approved the final version to be published and agreed to act as guarantors of the work.

Funding: This study was supported by an operating grant from Alberta Innovates - Health Solutions. Finlay McAlister and Sumit Majumdar hold career salary support from Alberta Innovates - Health Solutions. Finlay McAlister holds the Chair in Cardiovascular Outcomes Research at the Mazankowski Alberta Heart Institute, University of Alberta. Sumit Majumdar holds the Endowed Chair in Patient Health Management (supported by the Faculties of Medicine and Dentistry, and of Pharmacy and Pharmaceutical Sciences, University of Alberta). 